\title{
Low-Level-Laser-Therapy after Third Molar Removal in a Patient Affected by Osteogenesis Imperfecta
}

\section{Chiara Moreschi* and Stefano Bianchi}

Accademia Odontoiatrica Bianchi, Velletri Rome, Italy

*Corresponding Author: Chiara Moreschi, Accademia Odontoiatrica Bianchi, Velletri Rome, Italy.
Received: June 08, 2020

Published: June 30, 2020

(C) All rights are reserved by Chiara Moreschi and Stefano Bianchi.

\section{Abstract}

LLLT is used in oral surgery to reduce the discomfort of the patients after surgical procedures, such as pain, swelling and trismus.

We showed in our case report the use of LLLT as for the reduction of post-operative discomfort as to prevent osteonecrosis of the jaws, since our patient was affected with osteogenesis imperfecta.

The patient did not have any complications after surgery and after a one year follow-up she did not show any radiological or clinical sign of osteonecrosis of the jaws.

Keywords: Low Level Laser Therapy; Photo-Biomodulation; Oral Surgery; Third Lower Molar Extraction

\section{Introduction}

The extraction of third lower molars leads to some post-surgery complications such as pain, swelling and trismus, usually managed with drugs [1]. The use of the laser application at low intensity has been reported as useful in the control of the discomfort after third lower molar patient surgery [2] as in other surgical conditions such as nerve recovery after mandibular ramus osteotomy [2] or tissue healing after endodontic surgery [3].

In vitro study, trials demonstrated that LLLT enhanced growth and proliferation of human osteoblasts [4] and induced the expression of BMP-2, osteocalcin and TGF- $\beta 1$ [5]. Esmaeelinejad., et al. evaluated the effect of LLLT on human skin fibroblasts, he concluded that LLLT stimulated the release of IL 6 e bFGF from human fibroblasts [6].

In vivo studies showed that LLLT increased the bone healing and the wound healing $[7,8]$ and they demonstrated an increase in density and volume of trabecular bone $[9,10]$.
According to Matsumoto., et al. LLLT improved bone repair in rats that had undergone surgery and LLLT, using a mechanism of up-regulation for COX-2 expression in bone cells [11].

Our case report showed the use of LLLT in a patient, affected by osteogenesis imperfecta, with a prior therapy of bisphosphonate, who underwent third lower molar surgery. We used LLLT for the reduction of the discomfort of the patient after surgery, and for the prevention of osteonecrosis of the jaws.

\section{Case Report}

A 37 years old woman, affected by osteogenesis imperfecta, came to our observation in December 2018 with pain to the right retromolar region and swelling. After an X-Ray and clinical examination (Figure 1 and 2) a diagnosis of pericoronaritis of the right third lower molar was made and we decided for surgical therapy.

The patient has been affected by osteogenesis imperfecta since birth and she took bisphosphonate until June 2018.

Citation: Chiara Moreschi and Stefano Bianchi. "Low-Level-Laser-Therapy after Third Molar Removal in a Patient Affected by Osteogenesis Imperfecta". Acta Scientific Dental Sciences 4.7 (2020): 122-126. 


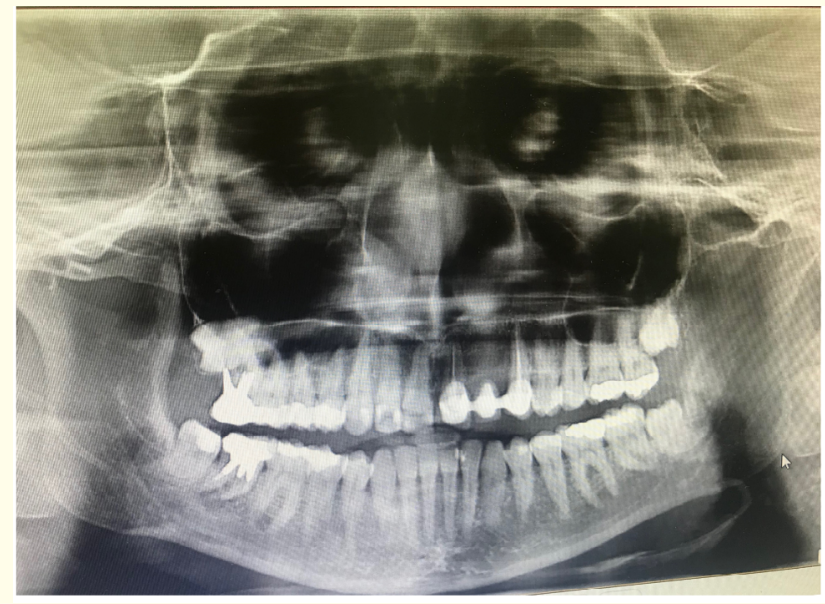

Figure 1: Pre-surgical X-Ray.

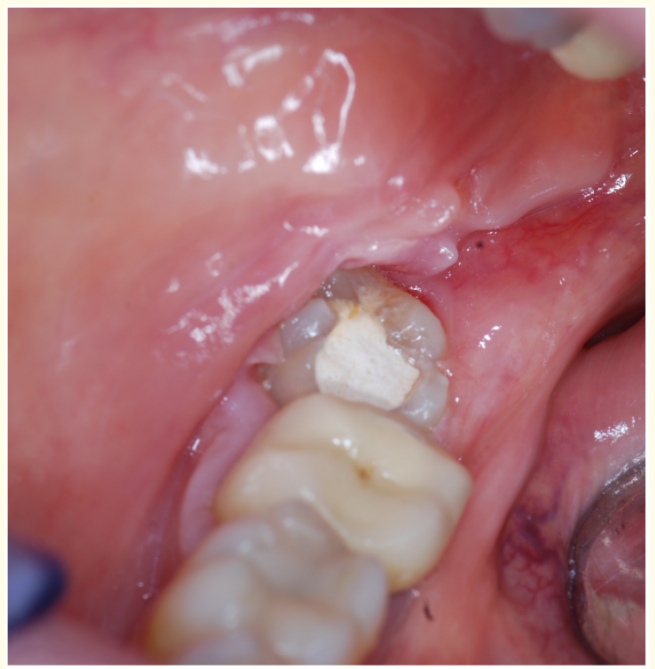

Figure 2: Image of intra-oral examination showing pericoronaritis of the right third lower molar.

The patient underwent surgery with the administration of the antibiotic therapy (Amoxicillin $2 \mathrm{gr} /$ die for 14 days). A local anesthesia was performed with articaine and 1:100.000 adrenaline. A triangular flap was made to expose the crown of the tooth (Figure 3), then the osteotomy was performed and the crown and the roots were separated and extracted (Figure 4). A silk 3-0 suture was positioned. We applied low-level laser therapy (Figure 5) with
Diode Laser Lasotronix Smart M PRO Fotona ${ }^{\circledR}$ (635nm wavelength, Energy density $3 \mathrm{~J} / \mathrm{cm}^{2}$, Beam area $2 \mathrm{~cm}^{2}$, Irradiation time $15 \mathrm{~s}$, Energy delivered $6 \mathrm{~J}$, Output Power $100 \mathrm{~mW}$, pulse rate continuous) to improve the healing of the wound once a week, until the healing mucosa covered the bone (Table 1).

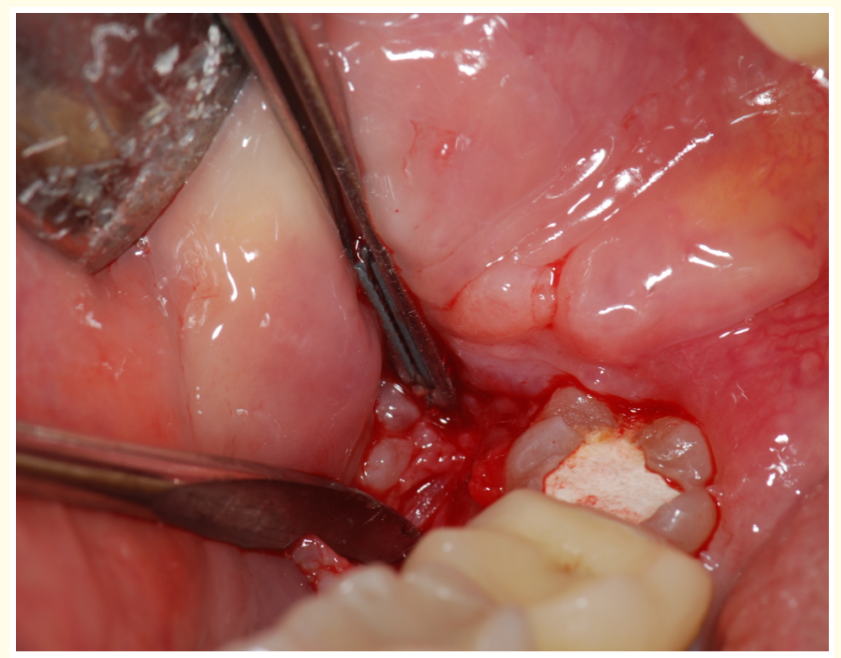

Figure 3: Image showing flap incision.

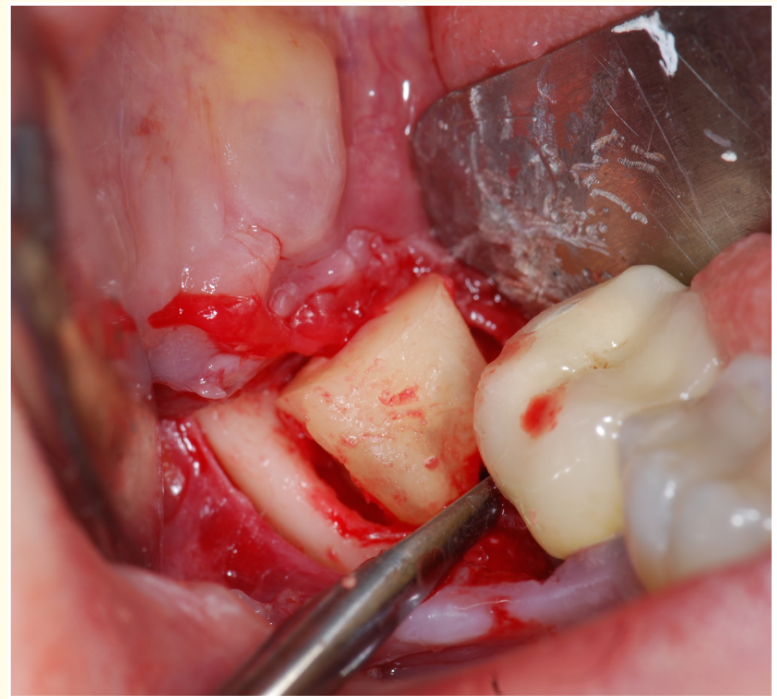

Figure 4: Image showing roots extraction. 


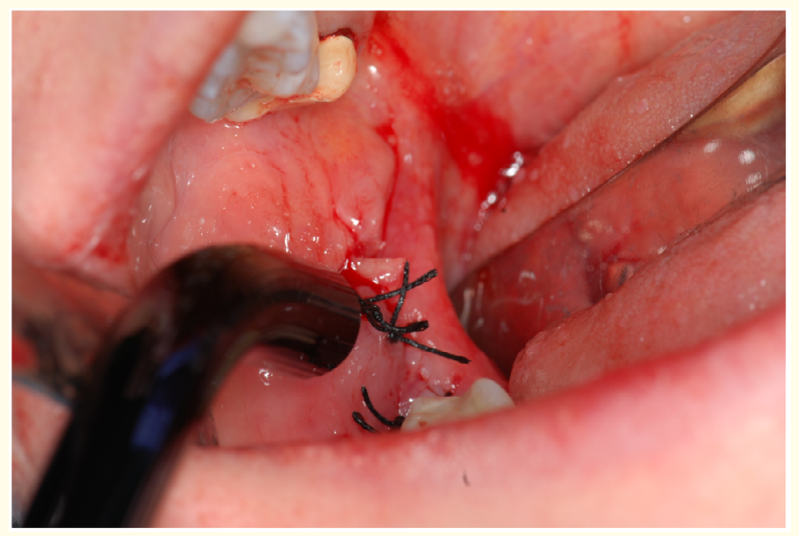

Figure 5: Image showing Low-Level laser therapy with Diode laser.

\begin{tabular}{|l|c|}
\hline \multicolumn{1}{|c|}{ Parameter } & Value \\
\hline Wavelength & $635 \mathrm{~nm}$ \\
\hline Beam Area & $2 \mathrm{~cm}^{2}$ \\
\hline Output Power & $100 \mathrm{~mW}$ \\
\hline Irradiation tIME & $15 \mathrm{~s}$ \\
\hline Energy Density & $3 \mathrm{~J} / \mathrm{cm}^{2}$ \\
\hline Energy Delivered & $6 \mathrm{~J}$ \\
\hline Pulse Rate & Continous \\
\hline
\end{tabular}

Table 1: Parameters used in this case report.

Home care instructions included antibiotics therapy, anti-inflammatory and analgesics therapy, $0.12 \%$ chlorhexidine mouth rinses twice a day for one week, ice therapy, liquid diet for two days. After 7 days of follow-up, the patient was healed without any complication and the suture was removed.

At one year follow-up the patient did not show any signs of osteonecrosis of the jaw (Figure 6).

\section{Discussion}

This case report demonstrated how the application of LLLT can be helpful in the management of postoperative discomfort after third molar surgery. In addition, our patient was affected by osteogenesis imperfecta and she took bisphosphonate until 2018, this could lead, in case of surgery, to a major risk to develop osteonecrosis of the jaws [12].

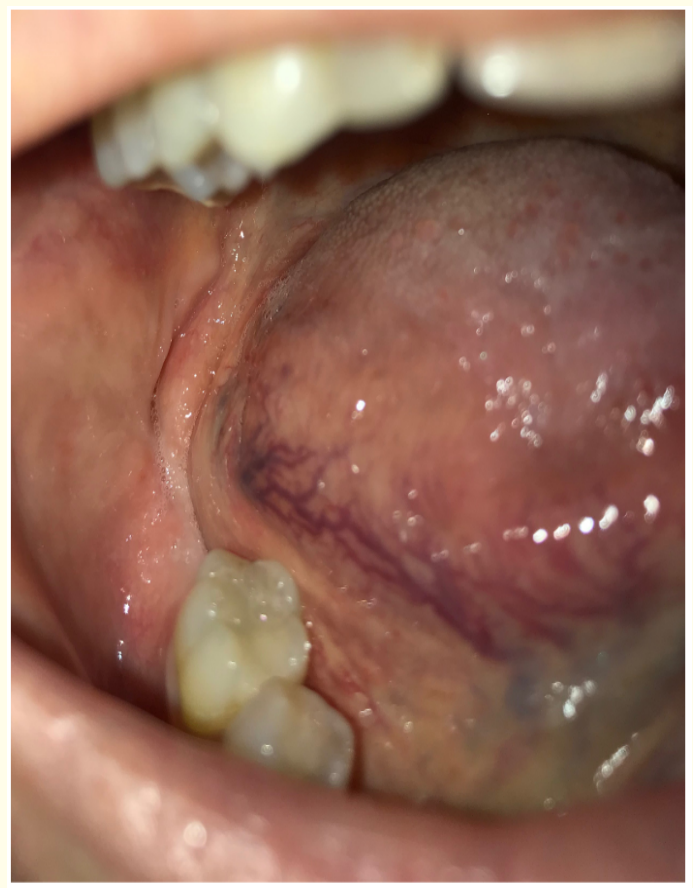

Figure 6: Image showing the one year follow-up with any clinical sign of osteonecrosis of the jaws.

According to Statkievicz., et al. the use of LLLT after tooth extraction, in patients with bisphosphonate therapy, can reduce the risk to develop osteonecrosis of the jaws [13].

Our patient did not have any complications and she did not report any discomfort during the post-operative period. At one year follow-up she had not developed any signs or symptoms of osteonecrosis of the jaws.

The extraction of an impacted third molar usually leads to some complications such as: pain, edema, trismus, limited jaw opening and movements [1]. Although, according to different authors in literature, LLLT can be helpful in reducing the post-operative discomfort [14-20], the efficacy of LLLT in prevention of pain, swelling and trismus is controversial. This is probably due to the different laser parameters, non-standardized protocol, different study design and difficulties in data analysis [21,22]. According to other authors LLLT, after third molar surgery, did not have any statistically significance in the reduction of pain and swelling when compared to the placebo site [23-27]. 
Dostolova., et al. analysed the effect of LLLT on inflammation associated with surgical removal of impacted lower third molar, using immunologic markers secretory immunoglobulin A and lysozyme. The healing process was evaluated with an infrared thermography. She concluded that with an $830 \mathrm{~nm}$ wavelength LLLT, a positive association between the immunoglobulin A and lysozyme was found, on the contrary there was no difference on the face temperature [28].

In a recent review Hosseinpour., et al. described LLLT as effective in different post operative conditions. The author concluded that LLLT had a positive effect in reducing pain, swelling and trismus after third molar tooth extraction, neurosensory and nerve recovery [29].

\section{Conclusion}

Our case report demonstrated that LLLT can be useful in the management of post-operative discomfort after lower third molar extraction. In addition we use LLLT as a prevention of osteonecrosis of the jaw in a patient with a previous therapy with bisphosphonate. Further studies are needed to confirm this report.

\section{Bibliography}

1. Candotto V., et al. "Complication in third molar extractions". Journal of Biological Regulators and Homeostatic Agents 33.3-1 (2019): 169-172.

2. Hosseinpour S., et al. "Photobiomodulation in Oral Surgery: A Review". Journal of Photobiomodulation, Photomedicine, and Laser 37.12 (2019): 814-825.

3. Metin R., et al. "Effects of low-level laser therapy on soft and hard tissue healing after endodontic surgery". Lasers in Medical Science 33.8 (2018): 1699-1706.

4. Stein E., et al. "Initial effects of low-level laser therapy on growth and differentiation of human osteoblast-like cells". Wiener Klinische Wochenschrift 120.3-4 (2008): 112-117.

5. Pyo SJ., et al. "Low-level laser therapy induces the expressions of BMP-2, osteocalcin, and TGF- $\beta 1$ in hypoxic-cultured human osteoblasts". Lasers in Medical Science 28.2 (2013): 543-550.

6. Esmaeelinejad M and Bayat M. "Effect of low-level laser therapy on the release of interleukin- 6 and basic fibroblast growth factor from cultured human skin fibroblasts in normal and high glucose mediums". Journal of Cosmetic and Laser Therapy 15.6 (2013): 310-317.

7. Noda M., et al. "High-frequency pulsed low-level diode laser therapy accelerates wound healing of tooth extraction socket: An in vivo study". Lasers in Surgery and Medicine 48.10 (2016): 955-964.

8. Park JB., et al. "Effects of increased low-level diode laser irradiation time on extraction socket healing in rats". Lasers in Medical Science 30.2 (2015): 719-726.

9. Hamad SA., et al. "Effect of Diode Laser on Healing of Tooth Extraction Socket: An Experimental Study in Rabbits". Journal of Oral and Maxillofacial Surgery 15.3 (2016): 308-314.

10. Kulkarni S., et al. "Efficacy of photobiomodulation on accelerating bone healing after tooth extraction: a systematic review". Lasers in Medical Science 34.4 (2019): 685-692.

11. Matsumoto MA., et al. "Low-level laser therapy modulates cyclo-oxygenase-2 expression during bone repair in rats". Lasers in Medical Science 24.2 (2009): 195-201.

12. Hayashi M., et al. "Risk of Delayed Healing of Tooth Extraction Wounds and Osteonecrosis of the Jaw among Patients Treated with Potential Immunosuppressive Drugs: A Retrospective Cohort Study". The Tohoku Journal of Experimental Medicine 246.4 (2018): 257-264.

13. Statkievicz C., et al. "Photomodulation multiple sessions as a promising preventive therapy for medication-related osteonecrosis of the jaws after tooth extraction in rats". The Journal of Photochemistry and Photobiology B: Biology 184 (2018): 7-17.

14. Landucci A., et al. "Efficacy of a single dose of low-level laser therapy in reducing pain, swelling, and trismus following third molar extraction surgery". International Journal of Oral and Maxillofacial Surgery 45.3 (2016): 392-398.

15. Kahraman SA., et al. "The Effects of Transcutaneous and Intraoral Low-Level Laser Therapy After Extraction of Lower Third Molars: A Randomized Single Blind, Placebo Controlled DualCenter Study". Photomedicine and Laser Surgery 35.8 (2017): 401-407.

16. Eshghpour M., et al. "Is Low-Level Laser Therapy Effective in the Management of Pain and Swelling After Mandibular Third 
Molar Surgery?" Journal of Oral and Maxillofacial Surgery 74.7 (2016): 1322.e1-1322.e13228.

17. Asutay F., et al. "Three-dimensional evaluation of the effect of low-level laser therapy on facial swelling after lower third molar surgery: A randomized, placebo-controlled study". Nigerian Journal of Clinical Practice 21.9 (2018): 1107-1113.

18. Merigo E., et al. "Efficacy of LLLT in swelling and pain control after the extraction of lower impacted third molars". Laser Therapies 24.1 (2015): 39-46.

19. Ferrante M., et al. "Effect of low-level laser therapy after extraction of impacted lower third molars". Lasers in Medical Science 28.3 (2013): 845-849.

20. Santos PL., et al. "Is Low-Level Laser Therapy Effective for Pain Control After the Surgical Removal of Unerupted Third Molars? A Randomized Trial". Journal of Oral and Maxillofacial Surgery 78.2 (2020): 184-189.

21. He WL., et al. "A systematic review and meta-analysis on the efficacy of low-level laser therapy in the management of complication after mandibular third molar surgery". Lasers in Medical Science 30.6 (2015): 1779-1788.

22. Noba C., et al. "Laser for bone healing after oral surgery: systematic review". Lasers in Medical Science 33.3 (2018): 667-674.

23. Pedreira AA., et al. "Thermographic and clinical evaluation of 808-nm laser photobiomodulation effects after third molar extraction". Minerva Stomatologica 65.4 (2016): 213-222.

24. Alan H., et al. "Evaluation of the effects of the low-level laser therapy on swelling, pain, and trismus after removal of impacted lower third molar". Head and Face Medicine 12.1 (2016): 25.

25. Tuk JGC., et al. "Analgesic effects of preinjection low-level laser/ light therapy (LLLT) before third molar surgery: a double-blind randomized controlled trial". Oral Surgery, Oral Medicine, Oral Pathology, and Oral Radiology 124.3 (2017): 240-247.

26. Eroglu CN and Keskin Tunc S. "Effectiveness of Single Session of Low-Level Laser Therapy with a $940 \mathrm{~nm}$ Wavelength Diode Laser on Pain, Swelling, and Trismus After Impacted Third Molar Surgery". Photomedicine and Laser Surgery 34.9 (2016): 406-410.
27. López-Ramírez M., et al. "Efficacy of low-level laser therapy in the management of pain, facial swelling, and postoperative trismus after a lower third molar extraction. A preliminary study". Lasers in Medical Science 27.3 (2012): 559-566.

28. Dostalova T., et al. "Low-Level Laser Therapy After Wisdom Teeth Surgery: Evaluation of Immunologic Markers (Secretory Immunoglobulin A and Lysozyme Levels) and Thermographic Examination: Placebo Controlled Study". Photomedicine and Laser Surgery 35.11 (2017): 616-621.

29. Hosseinpour S., et al. "Photobiomodulation in Oral Surgery: A Review". Photobiomodulation, Photomedicine, and Laser Surgery 37.12 (2019): 814-825.

\section{Assets from publication with us}

- Prompt Acknowledgement after receiving the article

- Thorough Double blinded peer review

- Rapid Publication

- Issue of Publication Certificate

- High visibility of your Published work

Website: www.actascientific.com/

Submit Article: www.actascientific.com/submission.php

Email us: editor@actascientific.com

Contact us: +919182824667 